\title{
Responsiveness and minimal clinically important difference for the EQ-5D in chronic rhinosinusitis*
}

Lloyd P. Hoehle 1,2 , Katie M. Phillips ${ }^{1,2}$, Marlene M. Speth³, David S. Caradonna ${ }^{1,4}$, Stacey T. Gray ${ }^{1,2}$, Ahmad R. Sedaghat 1,2,4,5

'Department of Otolaryngology, Harvard Medical School, Boston, MA, USA

2Department of Otolaryngology, Massachusetts Eye and Ear Infirmary, Boston, MA, USA

${ }^{3}$ Klinik für Hals-, Nasen-, Ohren- Krankheiten, Hals-und Gesichtschirurgie, Kantonsspital Aarau, Switzerland

${ }^{4}$ Division of Otolaryngology, Beth Israel Deaconess Medical Center, Boston, MA, USA

Departent of Otolaryngology and Communications Enhancement, Boston Children's Hospital, Boston, MA, USA
Rhinology 57: 2, 110 - 116, 2019

https://doi.org/10.4193/Rhin18.122

* Received for publication:

June 23, 2018

Accepted: July 24, 2018

Background: The 5-dimensional EuroQol questionnaire (EQ-5D) is validated to measure general health-related quality of life (QOL). Our objective was to determine the responsiveness and minimal clinically important difference (MCID) of the EQ-5D health utility value (EQ-5D HUV) and visual analog scale (EQ-5D VAS) in chronic rhinosinusitis (CRS).

Methods: 203 adults undergoing medical management for CRS were prospectively recruited. General health-related QOL (using EQ-5D HUV and EQ-5D VAS) and CRS-specific QOL (using the 22-item Sinonasal Outcome Test [SNOT-22]) were measured at enrollment and a subsequent follow-up time point 2-12 months later. At follow-up, participants also rated change in general health as "Much worse", "A little worse", "About the same", "A little better" or "Much better" compared to enrollment. The EQ-5D HUV and EQ-5D VAS MCIDs were calculated using distribution-based, anchor-based, and receiver operator characteristic (ROC) curve-based methods.

Results: Change in SNOT-22 score was correlated with EQ-5D HUV and EQ-5D VAS change. Using the different methods of calculating MCID, we find the EQ-5D HUV MCID to be 0.04 and EQ-5D VAS MCID to be 8.0. The calculated EQ-5D MCIDs had approximately a sensitivity of $40-50 \%$ and specificity of $80 \%$ in detecting patients experiencing noticeable improvement in general health.

Conclusions: The EQ-5D responds well to changing CRS symptomatology. We propose MCIDs for EQ-5D HUV of 0.04 and EQ-5D VAS of 8 in CRS patients, which although specific, are not sensitive for detecting patients experiencing improvement in general health.

Key words: chronic rhinosinusitis, minimal clinically important difference, minimum clinically important difference, MCID, 5-dimensional EuroQol questionnaire, EQ-5D, health utility value, visual analog scale, responsiveness

\section{Introduction}

Chronic rhinosinusitis (CRS) is an inflammatory disease of the paranasal sinuses that is present in up to $10 \%$ of the population $(1,2)$. The primary clinical manifestations of CRS include chronic symptomatology, acute sinonasal exacerbations, and exacerbations of comorbid pulmonary disease ${ }^{(3-8)}$. These clinical manifestations of the disease drive the downstream CRS disease consequences of decreased quality of life (QOL) and lost productivity. These disease consequences, in turn, lead to billions of dollars of costs annually for society due to direct healthcare expenses and indirect expenses ${ }^{(9)}$. The primary impact of CRS on patients is re- duced QOL and thus clinical decision-making is primarily made based on patient-reported outcome measures reflecting QOL. QOL impact can be assessed as disease-specific or general health-related QOL. CRS-specific quality of life may be quantified through numerous validated tools and questionnaires (1,2), including the 22-item Sinonasal Outcome Test (SNOT-22), which assesses nasal, sleep, ear/facial discomfort and emotional symptoms associated with CRS ${ }^{(10,11)}$. The SNOT-22 survey, for example, has been well validated for the assessment of CRS-specific QOL ${ }^{(10)}$. General health-related QOL is frequently assessed as one outcome metric for CRS ${ }^{(2)}$. The 5-dimensional 
EuroQol (EQ-5D) questionnaire is a validated tool for assessment of general health-related QOL using an associated health utility value (EQ-5D HUV) and visual analog scale (EQ-5D VAS) (12). The EQ-5D HUV assesses health status and ranges from 0 (lowest health state-death) to 1.0 (perfect health status) and is calculated from patient health status in five domains related to mobility, self care, usual activities, pain/discomfort, and anxiety/ depression. The EQ-5D VAS uses a visual analog scale to assesses patients' self-reported current state of health-with 0 as the minimum, representing the "worst health you can imagine", and 100 as the maximum, representing the "best health you can imagine". The EQ-5D has been previously used, validated and characterized for assessment of general health-related QOL and thus represents one important tool for studying CRS outcomes (13-15).

In the utilization of patient-reported outcome measures, the minimal clinically important difference (MCID) is frequently considered ${ }^{(16,17)}$. The MCID reflects the minimum change in a patientreported outcome measure that translates to a noticeable, and clinically meaningful, change for the patient. Characterization of the MCID for the SNOT-22, reportedly ranging from 9 to 12 , as well as an understanding of its accuracy to detect patients with noticeable improvement in their health after medical or surgical interventions for CRS has lead to many insights into the efficacy of CRS-related treatments ${ }^{(10,18)}$. The EQ-5D is increasingly used in the longitudinal assessment of general health-related QOL in CRS but no such characterization of its MCID has been performed. Previous studies of the MCID of the EQ-5D have shown different values for different disease processes ${ }^{(19-21)}$. Although the EQ-5D is a frequently used tool for assessment of general health-related QOL, its responsiveness and MCID for medically managed CRS patients has not been established. In this study, our objective was to establish the responsiveness and MCID of the EQ-5D HUV and EQ-5D VAS in a cohort of patients undergoing medical management of their CRS ${ }^{(22)}$.

\section{Materials and methods}

Study participants

This study was approved by our institution's Human Studies Committee. The participants in this study were adult patients aged 18 years or older with consensus guideline criteria established by the American Academy of Otolaryngology-Head and Neck Surgery for CRS ${ }^{(23)}$, and were recruited between August 1, 2016 and March 1, 2018 prospectively and provided informed consent for inclusion. Exclusion criteria included comorbid diagnoses of: 1) vasculitis, 2) cystic fibrosis, 3) sarcoidosis, and 4) immunodeficiency. In order to remove the confounding effect of recent endoscopic sinus surgery, patients who had endoscopic sinus surgery within the previous six months were also excluded from enrollment.

Study design and data collection
All patients who were enrolled were managed medically during the entire study period. As recommended by consensus guidelines/recommendations and supported by level 1 evidence, medical management of all participants' CRS included intranasal corticosteroids (spray or irrigations) and at least daily saline irrigation ${ }^{(2)}$. Short courses of systemic antibiotics or corticosteroids were provided as needed on a patient-specific basis and consistent with the recommended use of these medications for CRS patients ${ }^{(1,2)}$. Participants were assessed at two time pointsenrollment and the next follow-up visit, which was two to twelve months after enrollment. The length of time between enrollment and the next follow-up visit was determined on a patientby-patient basis but no participant underwent endoscopic sinus surgery during the study period.

At enrollment, the age, gender and smoking history of all participants were recorded. Any participant who reported current or former tobacco use was considered to be a smoker ${ }^{(24)}$. All participants completed a SNOT-22 as well as an EQ-5D from which both the EQ-5D HUV and EQ-5D VAS were determined ${ }^{(12,25)}$. All participants were also assessed by the evaluating rhinologist for a history of 1) aeroallergen hypersensitivity based on formal allergy testing, 2) asthma, and 3) nasal polyps at the time of enrollment based on nasal endoscopy. Aspirin sensitivity was determined based on a history of exacerbated airway symptoms with ingestion of non-steroidal anti-inflammatory drugs and a confirmatory provocation test with an oral challenge. At the next follow-up, participants completed another SNOT-22 and EQ-5D but also answered a question related to change in general health-related QOL that was previously used as an anchor for calculation of MCID in CRS patients undergoing endoscopic sinus surgery ${ }^{(10)}$. This anchor question asked participants to compare their general health at the follow-up visit to the time of enrollment (a "transition rating"), as previously described, on a five-item scale: "Much worse,, "A little worse", "About the same", "A little better", and "Much better" (10).

\section{Statistical analysis}

All analyses were performed with the statistical software package $R$ (www.r-project.org). Descriptive statistics, including use of unpaired t-test and ANOVA were performed. All correlations were calculated using Spearman's method. Regressions were performed as univariate linear regression where specified. Recruitment was performed in order to detect differences of large effect size $(\mathrm{d}=0.8)$ in the change of EQ-5D scores (HUV and VAS) in participants answering their anchor question as "About the same" compared to "A little better" with power of 0.8 at a significance level of 0.05 , reflecting an anchor-based method of MCID calculation. The EQ-5D MCIDs were calculated in three different ways. The first method was the distribution-based method that was calculated as half of the standard deviation of participants' EQ-5D scores at enrollment ${ }^{(26)}$. The second 
Table 1. Clinical and demographic characteristics of study participants.

\begin{tabular}{|lc|}
\hline & $\begin{array}{c}\text { All study } \\
\text { participants }\end{array}$ \\
\hline Demographics & $(\mathrm{N}=203)$ \\
\hline Age, mean in years, (SD) & $54.1(15.5)$ \\
\hline Gender & \\
\hline \multicolumn{1}{|c}{ Male } & $48.3 \%$ \\
\hline \multicolumn{1}{|c}{ Female } & $51.7 \%$ \\
\hline Smoking & $39.4 \%$ \\
\hline Comorbidities & \\
\hline Aeroallergen hypersensitivity & $48.8 \%$ \\
\hline Aspirin sensitivity & $4.9 \%$ \\
\hline Asthma & $32.0 \%$ \\
\hline CRS characteristics & \\
\hline Nasal polyps & $41.9 \%$ \\
\hline Previous sinus surgery & $37.4 \%$ \\
\hline Patient-reported outcome measures at & \\
\hline enrollment & \\
\hline SNOT-22 score, mean (SD) & $30.6(20.0)$ \\
\hline EQ-5D HUV, mean (SD) & $0.87(0.12)$ \\
\hline EQ-5D VAS, mean (SD) & $72.1(19.1)$ \\
\hline Patient-reported outcome measures at \\
follow up
\end{tabular}

method was an anchor-based method whereby the MCID was calculated as the difference in mean EQ-5D score (HUV and VAS) changes between participants responding with "About the same" compared to those responding with "A little better" (10,16,26). The third method for calculating MCID used Receiver Operator Characteristic (ROC) curve analysis ${ }^{(16)}$. The ROC method identified the change in SNOT-22 score which maximized the sum of sensitivity and specificity of identifying participants who reported an improvement ("A little better" or "Much better") in their sinus symptoms or general health. The area under the ROC curve (AUC) was calculated with the trapezoid rule and the $95 \%$ confidence interval of the AUC was calculated by performing 2000 bootstraps of the data.

\section{Results}

\section{Study participants}

There were a total of 203 participants and their baseline clinical and demographic characteristics are described in Table 1. The mean time between the baseline and follow-up visits was 170
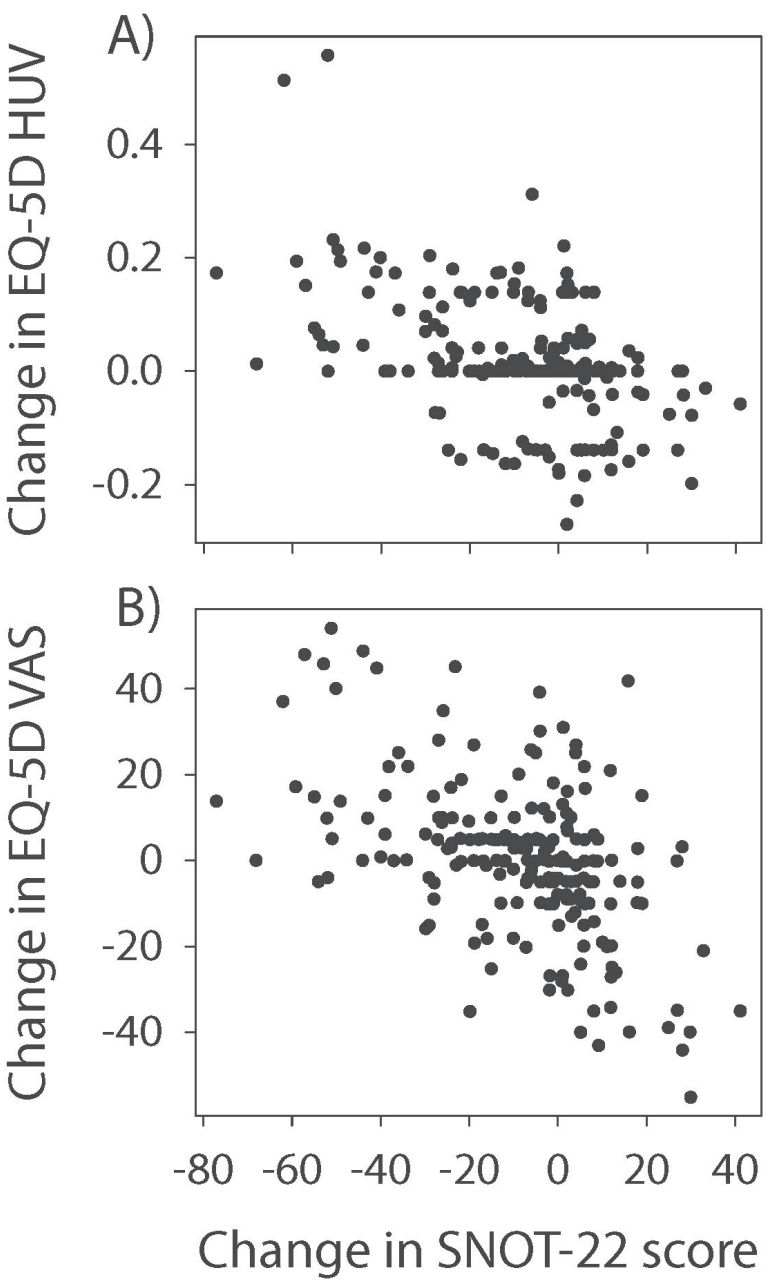

Figure 1. Scatterplot of change in participants' SNOT-22 scores vs. their change in A) EQ-5D HUV and B) EQ-5D VAS.

days (SD: 102 days; range: 62 - 364). The mean EQ-5D HUV was 0.85 (SD: 0.12; range: $0.35-1.00$ ) at enrollment and at 0.87 (SD: 0.12; range: $0.29-1.00$ ) at follow-up. The mean EQ-5D VAS was 71.8 (SD: 19.8; range: 10 - 100) at enrollment and 72.1 (SD: 19.1; range: 15 - 100) at follow-up. The mean change in EQ-5D HUV between study points was 0.02 (SD: 0.11 ; range: $-0.27-0.56$ ) and the mean change in EQ-5D VAS between study points was 0.3 (SD: 18.6; range: $-55-54$ ).

\section{Responsiveness of the EQ-5D}

We next studied the responsiveness of the EQ-5D by comparing the changes in EQ-5D HUV and EQ-5D VAS with the changes in SNOT-22 from enrollment to the follow-up time point (Figure 1). The change in the EQ-5D HUV was correlated with the change in SNOT-22 score ( $\rho=-0.42,95 \% \mathrm{Cl}$ : -0.53 to $-0.30, p<0.001)$. Every incremental increase in SNOT-22 score by one point was associated with a decrease of 0.003 in EQ-5D HUV (linear regression coefficient $[\beta]=-0.003,95 \% \mathrm{Cl}:-0.003$ to $-0.002, \mathrm{p}<0.001$ ). Likewise, the change in EQ-5D VAS was strongly correlated with 
Table 2. Anchor-based changes in EQ-5D.

\begin{tabular}{llcccc}
$\begin{array}{l}\text { General health anchor } \\
\text { transition ratings }\end{array}$ & Much worse & A little worse & About the same & A little better & Much better \\
\hline Change in EQ-5D HUV, mean (SD) & $-0.09(0.11)$ & $-0.01(0.10)$ & $0.0(0.09)$ & $0.04(0.09)$ & $0.08(0.15)$ \\
\hline Change in EQ-5D VAS, mean (SD) & $-8.7(28.6)$ & $-3.4(22.3)$ & $-4.6(15.0)$ & $2.9(15.4)$ & $12.6(18.0)$
\end{tabular}

the change in SNOT-22 score $(\rho=-0.47,95 \% \mathrm{Cl}$ : -0.57 to -0.35 , $\mathrm{p}<0.001)$. Every incremental increase in SNOT-22 score by one point was associated with a decrease of 0.5 in the EQ-5D VAS $(\beta=-0.5,95 \% \mathrm{Cl}:-0.6$ to $-0.3, \mathrm{p}<0.001)$.

\section{Determining the MCID of the EQ-5D HUV in medically managed CRS patients}

We first compared the changes in EQ-5D HUV values across all transition ratings represented in our general health anchor question (Figure 2 and Table 2 - top). There was a positive correlation between the transition ratings and the level of change in EQ-5D HUV ( $\rho=0.34,95 \% \mathrm{Cl}: 0.21-0.45, \mathrm{p}<0.001)$. Across the transition ratings of our anchor question, there were statistically significant differences in the calculated changes in EQ-5D HUV ( $p<0.001$ by ANOVA). Using our anchor question, we calculated the MCID of the EQ-5D HUV as the difference in the mean change of EQ-5D HUV between those reporting that their general health was "about the same" vs. "a little better", which we found to be 0.04 ( $95 \% \mathrm{Cl}$ : 0.01 to $0.08, \mathrm{p}=0.028$ ). An EQ-5D HUV MCID of 0.04 had $54.1 \%$ sensitivity and $79.8 \%$ specificity for detecting patients who experienced at least "a little" improvement in their general health. The ROC method, which seeks an MCID value that maximizes accuracy of identifying patients having improvement, found that an improvement in EQ-5D HUV of 0.01 ( $\mathrm{AUC}=0.687,95 \% \mathrm{Cl}: 0.611-0.764, \mathrm{p}<0.001$ ) maximized the sum of sensitivity (60.8\%) and specificity (73.6\%). These calculated MCIDs of 0.04 and 0.01 are in comparison to the distributionbased method of determining the EQ-5D HUV MCID, which was equal to 0.06 (one half the standard deviation of participants' EQ-5D HUV at enrollment).

\section{Determining the MCID of the EQ-5D VAS in medically managed CRS patients}

We repeated the above analyses to determine an MCID for the EQ-5D VAS. Comparing the changes in EQ-5D VAS values across all transition ratings, represented in our general health anchor question (Figure 3 and Table 2 - bottom), we found a positive correlation between the transition ratings and the level of change in EQ-5D VAS ( $\rho=0.31,95 \% \mathrm{Cl}: 0.18-0.43, p<0.001)$. Across the transition ratings of our general health anchor question, there were statistically significant differences in the calculated changes in EQ-5D VAS ( $p<0.001$ by ANOVA). The difference in the mean change of EQ-5D VAS between those reporting that their general health was "about the same" vs. "a little better" resulted in an MCID of 7.5 (95\%Cl: 1.1 to 14.0, p=0.023). An MCID of 7.5 for the EQ-5D VAS had $40.5 \%$ sensitivity and $81.4 \%$ specificity for detecting patients who experienced at least "a little" improvement in their general health. The ROC method identified an improvement in EQ-5D VAS of 1.0 ( $\mathrm{AUC}=0.707,95 \% \mathrm{Cl}$ : $0.634-$ $0.780, p<0.001)$ which maximized the sum of sensitivity $(67.6 \%)$ and specificity (66.7\%). These calculated MCIDs of 7.5 and 1.0 are in comparison to the distribution-based method of determining the EQ-5D VAS MCID, which was equal to 9.9.

\section{Discussion}

CRS is characterized by a significant decrease in a patient's QOL $(1,2)$. Using general health-related QOL, a metric of QOL that is comparable across different diseases ${ }^{(12,27)}$, it has been shown that the QOL detriment associated with CRS is worse than or similar to other serious, chronic diseases such as diabetes, asthma and heart disease ${ }^{(2)}$. General health-related QOL is also an important metric since cost-effectiveness can be determined by weighing the cost of an intervention against the improvement in general health-related QOL that it provides ${ }^{(28)}$. For example, prior study has established the cost-effectiveness of endoscopic sinus surgery for chronic rhinosinusitis by comparing post-surgical improvements of general health-related $\mathrm{QOL}$ to the costs associated with the surgery ${ }^{(29)}$. The EQ-5D is one tool for measuring general health-related QOL that has been utilized in the study of many diseases, including CRS ${ }^{(13,25)}$. Prior studies of the EQ-5D have validated both the EQ-5D HUV and EQ-5D VAS -including the establishment of responsiveness and MCID_for a number of diseases. However, despite the use of the EQ-5D for the study of patients with CRS - a disease that is predominantly managed medically - the responsiveness of the EQ-5D for medically managed CRS patients is unknown. Moreover, the MCID of the EQ-5D HUV and EQ-5D VAS have never been established for any CRS patients. Here we demonstrate the responsiveness of the EQ-5D as a metric for general health-related QOL measurement for medically managed CRS patients, and based on the results we have presented, we propose the MCID of EQ-5D HUV to be 0.04 and the MCID of the EQ-5D VAS to be 8 for patients with CRS.

The EQ-5D, consisting of two components, was developed as 


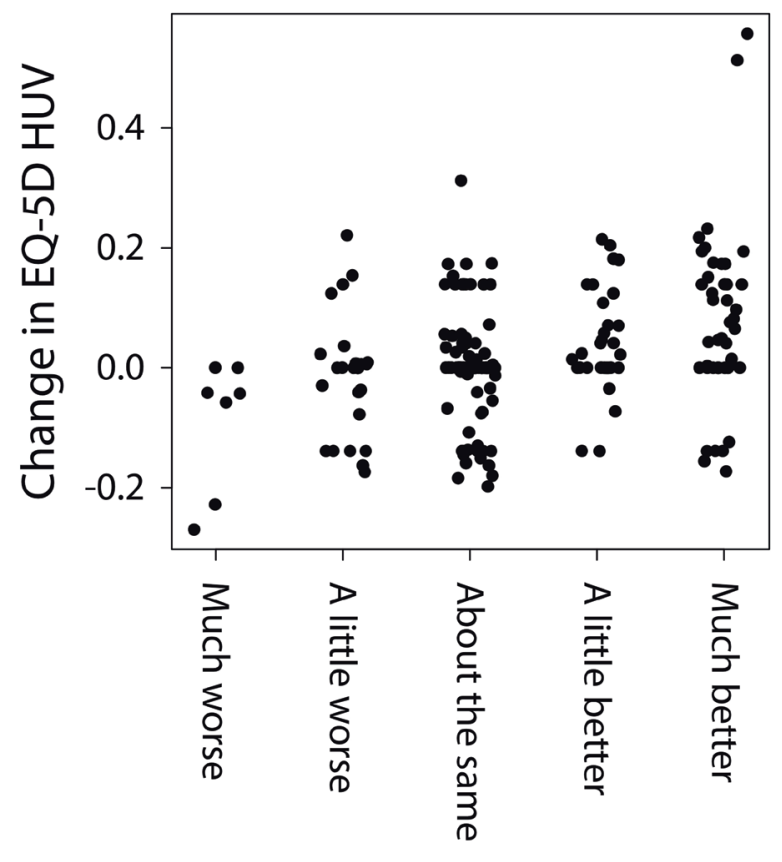

General health anchor response

Figure 2. Scatterplot of change in EQ-5D HUV against participant responses to the general health anchor question.

a means of measuring general health-related $\mathrm{QOL}{ }^{(25)}$. The first component consists of questions in five domains related to mobility, self-care, usual activities, pain/discomfort and anxiety/ depression, the answers to which can be used to calculate the EQ-5D HUV. The second component is an unbiased measurement of general health-related QOL (i.e. does not specifically ask about any particular problem) using a VAS that asks patients to simply rate "how good or bad your health is TODAY" on a scale of 0 (representing the worst health imaginable) to 100 (representing the best health imaginable) ${ }^{(25)}$. Since it was developed, the EQ-5D has been used for measuring general health-related QOL for many diseases as well as determining quality-adjusted life years imparted by treatments in cost-effectiveness calculations (28). Previous studies have identified the MCID of the EQ-5D HUV to range between 0.05 to $0.54{ }^{(19)}$ and the MCID of the EQ-5D VAS to range between 6 to 8 for diseases not including CRS $(20,30)$. In order to establish its validity for a specific disease, it is important to establish both responsiveness and MCID for the EQ-5D in the setting of that disease ${ }^{(22)}$. Our findings not only establish the responsiveness of the EQ-5D for CRS patients but our results are also largely consistent with previous studies reporting MCID for EQ-5D HUV and EQ-5D VAS, although there is clearly variability in MCID from disease to disease. MCID is an important concept in the use of patient-reported outcome measures and was first defined as "the smallest difference in score in the domain of interest, which patients perceive as beneficial and which

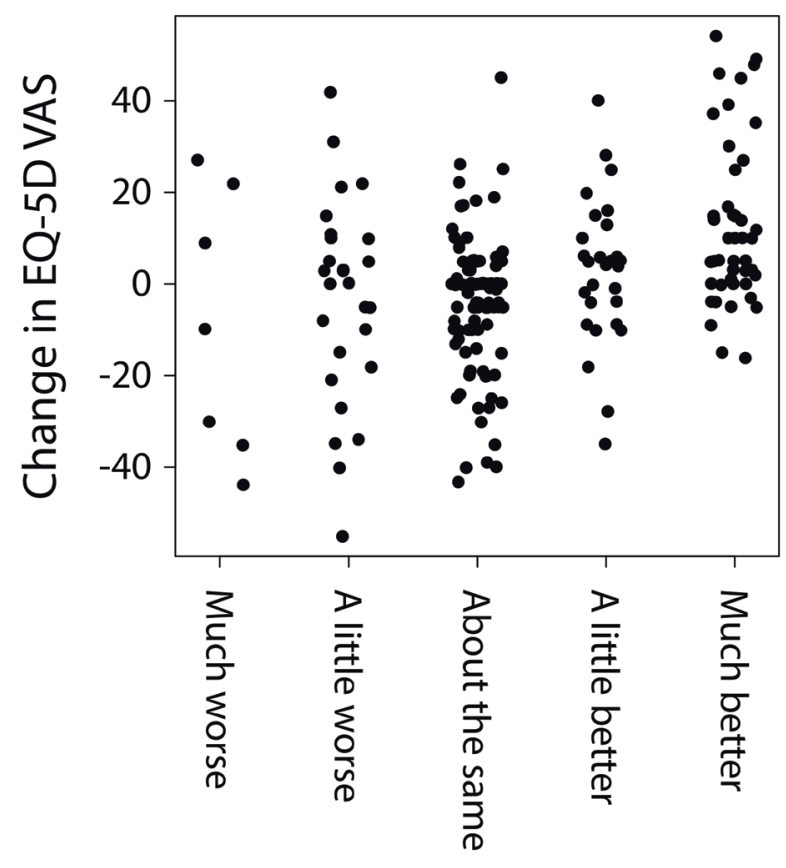

General health anchor response

Figure 3. Scatterplot of change in EQ-5D VAS against participant responses to the general health anchor question.

would mandate, in the absence of troublesome side effects and excessive cost, a change in the patient's management." ${ }^{(31)}$. Since patient-reported outcome measures are subjective and not made based on objective benchmarks, there is some inherent intra-individual variability in how they are reported. As a result, it may be unclear how much of an improvement in a patient-reported outcome measure may translate to a clinically meaningful change. This may be a particularly important consideration in the interpretation of improved patient-reported outcome measures after application of a treatment, e.g. as might be reported for a clinical trial. The MCID has been proposed as one means of determining a threshold for a significant or meaningful change in a patient-reported outcome measure ${ }^{(31)}$. However, the MCID is also affected by patient biases including the context (e.g. disease state or treatment status) within which the MCID is calculated ${ }^{(32,33)}$. The utilization of MCID therefore requires calculation in specific disease scenarios. As the EQ-5D is increasingly being used in studies of general health-related QOL in CRS patients, we believe that our results may serve as a benchmark against which results of studies using the EQ-5D for CRS patients may be compared.

However, a strict application of the MCID in interpreting treatment effect sizes should be done with the limitations of the MCID in mind. Previous studies of MCIDs have often found them to be specific for identifying patients with clinically significant improvement but poorly sensitive ${ }^{(34-36)}$. In other words, a not 
insignificant fraction of patients experiencing a clinically significant improvement does not necessarily exceed an MCID of improvement in the corresponding patient-reported outcome measures. This is also consistent with our study, which found the MCID of the EQ-HUV (0.04) to have $79.8 \%$ specificity but only $54.1 \%$ sensitivity in detecting CRS patients with noticeable improvement in general health-related QOL while the MCID of the EQ-5D VAS ${ }^{(8)}$ had $81.4 \%$ specificity but only $40.5 \%$ sensitivity. We have also found the same to be true for the MCID of the SNOT22 , which we found to be highly specific (80-90\%) but poorly sensitive (50-60\%) in detecting CRS patients who experienced a noticeable improvement in sinonasal symptom burden ${ }^{(18)}$. Nevertheless, the results of our present study, while providing a context within which future EQ-5D results in studies of CRS patients may be placed, show that universal and strict application of the MCID may not be accurate in identifying all clinically significant improvement in general health-related QOL. Instead, these results further support the notion that MCID should be applied judiciously ${ }^{(32,33)}$ with an understanding of its sensitivity and specificity in detecting patients experiencing clinically significant and meaningful improvement in a patient-reported outcome measure.

This study should be interpreted in the context of its limitations and, in particular, the limitations of the MCID. One important consideration in any calculated MCID is how an anchor question was applied: how much improvement is the minimum clinically important improvement-_a little" or "a lot"? Consistent with prior work on CRS patients, we deemed at least "a little" improvement in patient-reported general health to represent the minimal clinically important improvement ${ }^{(10,18)}$. We acknowledge that this may be a point of debate, an area of future investigation and yet another reflection of the subtleties that are incorporated into the calculation (and must be considered in the interpretation) of MCID. General health-related QOL may also be affected by disease processes that are distinct from the prototypical symptomatic manifestations of CRS, for example acute exacerbations of CRS or its effects on comorbid asthma $(4,5,8,37)$. Beyond CRS-specific disease manifestations, it is possible that the status of other diseases may also impact a CRS patient's general health-related QOL. Thus changes in EQ-5D could be driven by many CRS-specific or CRS-independent disease processes. Additionally, none of these patients underwent endoscopic sinus surgery, therefore it is certainly possible that the EQ-5D MCIDs may be different in surgically managed CRS patients ${ }^{(10,18)}$. In fact, MCID could potentially be different in different subsets of patients, for example those patients with polyps vs. those patients without polyps. Finally, our patients were all medically managed with saline irrigations and intranasal corticosteroid sprays. The use of adjunctive systemic antibiotics or corticosteroids, which were given on a patient-by-patient basis, may have introduced a confounding effect in EQ-5D responsiveness or MCID, although we specifically chose a minimum 2-month follow-up time period to maximize the likelihood of wash-out of any systemic effects of such prescribed medications.

\section{Acknowledgement}

No funding was used to support this study. The authors gratefully acknowledge the contribution of the patients who participated in this study.

\section{Authorship contribution}

LPH, KMP, MMS, DSC, STG: performed the study and wrote/revised the manuscript. ARS: conceived, designed, and performed the study and wrote/revised the manuscript.

\section{Conflict of interest}

The authors declare no conflicts of interest.

\section{References}

1. Fokkens WJ, Lund VJ, Mullol J, Bachert C, Alobid I, Baroody F, et al. European Position Paper on Rhinosinusitis and Nasal Polyps 2012. Rhinol Suppl 2012 Mar;(23)(23):298.

2. Orlandi RR, Kingdom TT, Hwang PH, Smith $\mathrm{TL}$, Alt JA, Baroody FM, et al. International Consensus Statement on Allergy and Rhinology: Rhinosinusitis. Int Forum Allergy Rhinol 2016 Feb;6 Suppl 1:S209.

3. Hoehle LP, Phillips KM, Bergmark RW, Caradonna DS, Gray ST, Sedaghat AR. Symptoms of chronic rhinosinusitis differentially impact general health-related quality of life. Rhinology 2016 Sep 25;54(4):316322.

4. Banoub RG, Phillips KM, Hoehle LP, Caradonna DS, Gray ST, Sedaghat AR. Relationship between chronic rhinosi- nusitis exacerbation frequency and asthma control. Laryngoscope 2018 May 01;128(5):1033-1038.

5. Phillips KM, Hoehle LP, Caradonna DS, Gray ST, Sedaghat AR. Association of severity of chronic rhinosinusitis with degree of comorbid asthma control. Ann Allergy Asthma Immunol 2016 Dec;117(6):651-654.

6. Campbell AP, Phillips KM, Hoehle LP, Feng AL, Bergmark RW, Caradonna DS, et al. Depression symptoms and lost productivity in chronic rhinosinusitis. Ann Allergy Asthma Immunol 2017 March 01;118(3):286-289.

7. Speth MM, Hoehle LP, Phillips KM, Caradonna DS, Gray ST, Sedaghat AR. Changes in chronic rhinosinusitis symptoms differentially associate with improvement in general health-related quality of life. Ann Allergy Asthma Immunol 2018, Aug;121(2):195-199.

8. Phillips KM, Hoehle LP, Bergmark RW, Caradonna DS, Gray ST, Sedaghat AR. Acute Exacerbations Mediate Quality of Life Impairment in Chronic Rhinosinusitis. J Allergy Clin Immunol Pract 2017 April 01;5(2):422-426.

9. Rudmik L. Economics of Chronic Rhinosinusitis. Curr Allergy Asthma Rep 2017 April 01;17(4):5.

10. Hopkins C, Gillett S, Slack R, Lund VJ, Browne JP. Psychometric validity of the 22-item Sinonasal Outcome Test. Clin Otolaryngol 2009 Oct;34(5):447-454.

11. Feng AL, Wesely NC, Hoehle LP, Phillips KM, Yamasaki A, Campbell AP, et al. A validated model for the 22-item Sino-Nasal Outcome Test subdomain structure in chronic rhi- 
nosinusitis. Int Forum Allergy Rhinol 2017 Dec;7(12):1140-1148.

12. EuroQol Group. EuroQol--a new facility for the measurement of health-related quality of life. Health Policy 1990 Dec;16(3):199-208.

13. Remenschneider AK, D'Amico L, Gray ST, Holbrook EH, Gliklich RE, Metson R. The EQ-5D: a new tool for studying clinical outcomes in chronic rhinosinusitis. Laryngoscope 2015 Jan;125(1):7-15.

14. Bewick J, Morris S, Hopkins C, Erskine S, Philpott CM. Health utility reporting in chronic rhinosinusitis patients. Clin Otolaryngol 2018, Feb;43(1):90-95.

15. Yamasaki A, Hoehle LP, Phillips KM, Feng AL, Campbell AP, Caradonna DS, et al. Association between systemic antibiotic and corticosteroid use for chronic rhinosinusitis and quality of life. Laryngoscope 2018 Jan;128(1):37-42.

16. Gerlinger C, Schmelter T. Determining the non-inferiority margin for patient reported outcomes. Pharm Stat 2011 Oct;10(5):410413.

17. Jayadevappa R, Cook R, Chhatre S. Minimal important difference to infer changes in health-related quality of life-a systematic review. J Clin Epidemiol 2017 Sept;89:188198.

18. Phillips KM, Hoehle LP, Caradonna DS, Gray ST, Sedaghat AR. Minimal clinically important difference for the 22-item Sinonasal Outcome Test in medically managed patients with chronic rhinosinusitis. Clin Otolaryngol 2018 June 28. [Epub ahead of print]

19. Coretti S, Ruggeri M, McNamee P. The minimum clinically important difference for EQ-5D index: a critical review. Expert Rev Pharmacoecon Outcomes Res 2014 Apr;14(2):221-233.

20. Zanini A, Aiello M, Adamo D, Casale S, Cherubino F, Della Patrona S, et al. Estimation of minimal clinically important difference in EQ-5D visual analog scale score after pulmonary rehabilitation in subjects with COPD. Respir Care 2015 Jan;60(1):88-95

21. Caplan N, Robson H, Robson A, Barry G, Wilkes $G$. Associations between community-based physiotherapy for musculoskeletal injury and health related quality of life (EQ5D): a multi-centre retrospective analysis Health Qual Life Outcomes 2017 Oct;15(1):3.

22. Payakachat N, Ali MM, Tilford JM. Can The EQ-5D Detect Meaningful Change? A Systematic Review. Pharmacoeconomics 2015 Nov;33(11):1137-1154.

23. Rosenfeld RM, Piccirillo JF, Chandrasekhar SS, Brook I, Ashok Kumar K, Kramper M, et al. Clinical practice guideline (update): adult sinusitis. Otolaryngol Head Neck Surg 2015 Apr;152(2 Suppl):S39.

24. Phillips KM, Hoehle L, Bergmark RW, Caradonna DS, Gray ST, Sedaghat AR. Reversal of Smoking Effects on Chronic Rhinosinusitis after Smoking Cessation. Otolaryngol Head Neck Surg 2017 Oct; 157(4):737-742.

25. Devlin NJ, Brooks R. EQ-5D and the EuroQol Group: Past, Present and Future. Appl Health Econ Health Policy 2017 Apr;15(2):127-137.

26. Cook CE. Clinimetrics Corner: The Minimal Clinically Important Change Score (MCID): A Necessary Pretense. J Man Manip Ther 2008;16(4):82

27. Fryback DG, Dunham NC, Palta $M$, Hanmer J, Buechner J, Cherepanov D, et al. US norms for six generic health-related quality-of-life indexes from the National Health Measurement study. Med Care 2007 Dec;45(12):1162-1170.

28. Stamuli E. Health outcomes in economic evaluation: who should value health? Br Med Bull 2011:97:197-210.

29. Rudmik L, Soler ZM, Mace JC, Schlosser RJ, Smith TL. Economic evaluation of endoscopic sinus surgery versus continued medical therapy for refractory chronic rhinosinusitis. Laryngoscope 2015 Jan;125(1):2532

30. Nolan CM, Longworth L, Lord J, Canavan $J \mathrm{~L}$, Jones SE, Kon SS, et al. The EQ-5D-5L health status questionnaire in COPD: validity, responsiveness and minimum important difference. Thorax 2016 Jun;71 (6):493-500.

31. Jaeschke R, Singer J, Guyatt GH. Measurement of health status. Ascertaining the minimal clinically important difference. Control Clin Trials 1989 Dec;10(4):407-415.

32. Wright A, Hannon J, Hegedus EJ, Kavchak
AE. Clinimetrics corner: a closer look at the minimal clinically important difference (MCID). J Man Manip Ther 2012 Aug;20(3):160-166.

33. Jones PW, Beeh KM, Chapman KR, Decramer M, Mahler DA, Wedzicha JA. Minimal clinically important differences in pharmacological trials. Am J Respir Crit Care Med 2014 Feb;189(3):250-255.

34. Hui D, Shamieh O, Paiva CE, Perez-Cruz PE, Kwon JH, Muckaden MA, et al. Minimal clinically important differences in the Edmonton Symptom Assessment Scale in cancer patients: A prospective, multicenter study. Cancer 2015 Sep;121(17):3027-3035.

35. Young VN, Jeong $K$, Rothenberger SD, Gillespie Al, Smith LJ, Gartner-Schmidt JL, et al. Minimal clinically important difference of voice handicap index-10 in vocal fold paralysis. Laryngoscope. 2018 Jun;128(6):14191424.

36. Amaral R, Carneiro AC, Wandalsen G, Fonseca JA, Sole D. Control of Allergic Rhinitis and Asthma Test for Children (CARATKids): Validation in Brazil and cutoff values. Ann Allergy Asthma Immunol 2017 May;118(5):556.e2

37. Phillips KM, Hoehle LP, Bergmark RW, Campbell AP, Caradonna DS, Gray ST, et al. Chronic rhinosinusitis severity is associated with need for asthma-related systemic corticosteroids. Rhinology. $2017 \mathrm{Sep}$ 1;55(3):211-217.

Ahmad R. Sedaghat, MD, PhD

Department of Otolaryngology Massachusetts Eye and Ear Infirmary

243 Charles Street

Boston, MA

USA 02114

Tel: +001 617-573-6011

Fax: +001 617-573-6845

E-mail:

ahmad_sedaghat@meei.harvard.edu 